\title{
Design and Analysis of Model Predictive Control based Direct and Indirect Current strategy for Single Phase Shunt Active Power Filter
}

\author{
Rameshkumar Kanagavel ${ }^{1 *}$, Indragandhi $\mathrm{V}^{2}$ and Palanisamy $\mathrm{K}^{2}$ \\ ${ }^{1}$ Sri Krishna College of Engineering and Technology, Coimbatore, India. \& Research Scholar, VIT \\ University, Vellore, Tamilnadu, India \\ ${ }^{2}$ VIT University, Vellore, Tamilnadu, India
}

\begin{abstract}
In this paper presents a comparative analysis of two control method applied to a single phase Shunt Active Power Filter (SAPF). It is about Model Predictive Control (MPC) based Direct Current Control (DCC) and Indirect Current Control (IDCC) strategy. The performances of two current control strategies were verified through a simulation with MATLABSimulink Software. Simulation results confirmed that compared to the DCC strategy, the IDCC strategy using MPC becomes simpler and need less hardware components.
\end{abstract}

\section{Introduction}

In recent years, using Non Linear Loads (NLLs) causes a significant power quality problem in power distribution networks. The NLLs such as electric furnaces, light controllers, solid-state ac voltage regulators, temperature controllers, and uninterruptable power supplies cause problems in power systems and consumer products. Problems may be: mal-operation of some protective equipment, overheating of the transformer, power factor correction capacitor, machine vibrations, capacitor fuses blown, inaccurate power metering, and disturbance to nearby communication networks [1-4].

To avoid the above mentioned adverse effects and improve the power quality, traditional solutions such as passive filters have been used for their simplicity, low cost, and efficiency, but they are ineffective due to the drawbacks of their huge size, fixed compensation and may cause series or parallel resonance problems. Also, the passive filters cannot adjust to the system characteristic changes. In order to overcome those problems the most effective method of improving power quality the use of Shunt Active Power Filter (SAPF) is preferred. The effective operation of a shunt active power filter mainly depends upon two areas. The first one is used to estimate the reference current and the second one is type of current controller used for generation of gate signals for power switching devices of voltage source inverter. Various current control methods are used to improve the SAPF performance. One of the most popular methods for current control in SAPF is MPC control technique [5-7]. MPC was developed in 1970 in process control industry, the interest in it has reached its pinnacle only in

*Corresponding author: rameshvel.k@gmail.com 
recent days. This method is based on a simple concept, and easy to implement in real-time due to the fast development of controller such as microprocessors and digital signal processors can handle such computations [8].

This manuscript aims to analysis of two control method applied to a single phase SAPF. It is about MPC based Direct Current Control (DCC) and Indirect Current Control (IDCC) strategy. This manuscript is offered in three parts. Initially starting with an introduction, the following sections deal with the control of single phase SAPF, Experimental results and finally end with suitable conclusion.

\section{SAPF Control strategy}

The control scheme of single phase shunt active filter is the important portion for its effective performance in order to eliminate the harmonics and which can be mainly implemented over and done with in two steps,

- Extraction of reference compensating current

- Generation of gate signals for power switching devices of voltage source inverter.

Generally many number of reference current extraction algorithm are used to derive the reference current signal for the control of SAPF. Mainly which are classified into two types, such as time domain and frequency domain based control techniques. Time domain techniques are based on instantaneous approximation of reference compensating signal in the form of current and voltage signals from biased and harmonically polluted current and voltage signals. These methods are applicable for both single phase and three phase systems. The frequency domain technique is based on the Fourier analysis of distorted current and voltage signals to extract the reference compensating signals. Fourier transformation are used to separate the harmonically polluted current and voltage signals and combined together to generate the reference compensating signal.

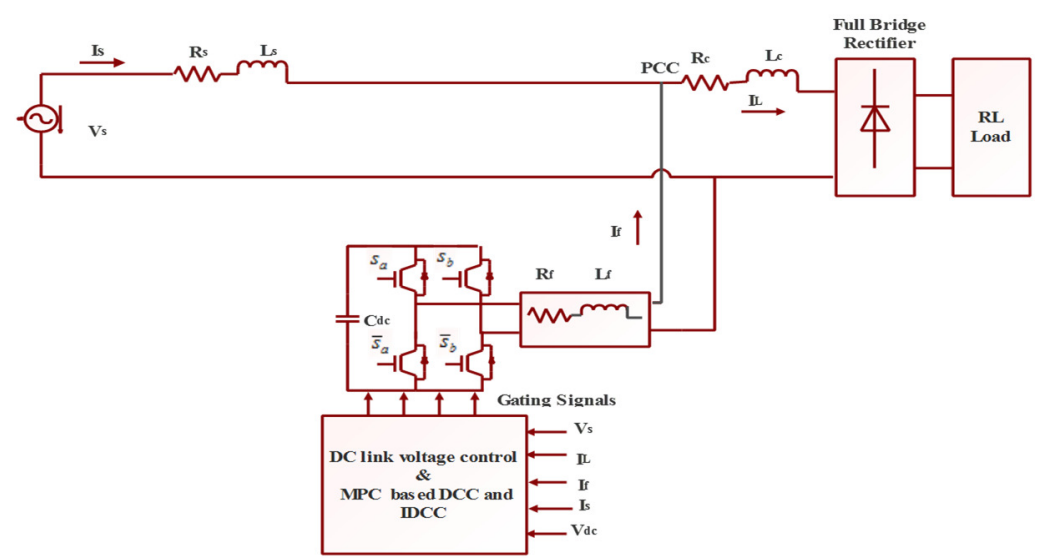

Fig.1. Single phase SAPF Model

In this paper DC link voltage control based reference current extraction technique is been used. This scheme contains regulation of the dc link voltage to set the amplitude of reference current for harmonic and reactive power compensation. Initially the capacitor voltage is compared with a reference value and fed to a PI controller. The output of PI controller is taken as the peak value of reference source current (Isp, ref). Then output of PI controller is multiplied by a unity amplitude of sine wave to obtain the sinusoidal reference source current. Finally by comparing the measured supply current with sinusoidal reference source current the gating signals for SAPF is generated. In the above strategy which is generally composed 
of two control loops. An outer voltage loop is used to regulate the DC-link voltage while an inner current loop is used for regulating the filter phase current.

\subsection{Model Predictive Current Control}

There are several current control methods are used to improve the SAPF performance. One of the most popular methods for current control in SAPF is MPC control technique. MPC was developed in 1970 in process control industry, the interest in it has reached its pinnacle only in recent days. The main advantages of the MPC controlled inverters are the absence of modulator, utilization of variable switching frequency, the possibility of online optimization, lower complexity and the inclusion of constraints. Rameshkumar et al. [8] uses a MPCC in single phase SAPF and analysed with various loads using matlab simulation. Fig.2.a and Fig. 2.b shows the schematic diagram of an MPC based single phase SAPF. The primary control of MPC control is to minimize the error between the references and predicted load current which is defined as cost function or quality function. To attain this objective, the SAPF switching state which is going to reduces ' $\mathrm{g}$ ' is choose and given at the time of next sampling instant.

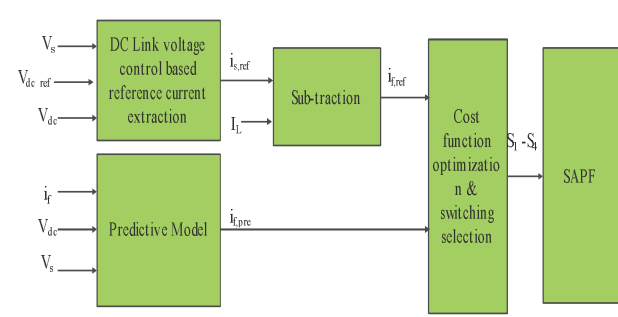

Fig. 2a. MPC based DCC

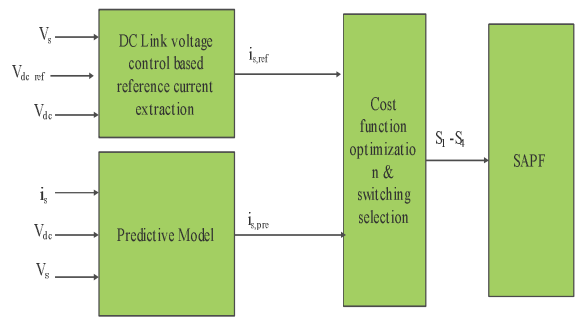

Fig. 2b. MPC based IDCC

The Model predictive current control of SAPF is mainly classified in to two types.

\section{Model predictive control based direct current control (reference compensating current as reference filter current).}

In MPC based DCC scheme the switching signals for the inverter are obtained by comparing actual SAPF current (injection current) with its non-sinusoidal reference current. the non-sinusoidal reference current as a reference shunt SAPF current are obtained by subtracting sinusoidal reference source current from load current as shown in Fig.2.a. The concept of design and calculation of model predictive control based direct current control used by the authors of (8) to simulate the MPC based SAPF control. According to [8] the equation for the possible predicted output current can be expressed as follows:

$$
i_{f, p r e}(k+1)=\frac{T_{s}}{L_{e q}}\left(V_{i}(k)-V_{s}(k)\right)+\left(1-\frac{R_{e q} T_{s}}{L_{e q}}\right) i_{f}(k)
$$

So as to apply optimal switching signal to the SAPF, the all possible predicted filter currents are compare with filter reference current using cost function ' $\mathrm{g}$ '. It can as represented as

$$
g=\left(i_{f, r e f}(k+1)-i_{f, p r e}(k+1)\right)
$$


In ICC scheme the switching signals for the inverter are obtained by comparing the measured supply current with sinusoidal reference current as shown in Fig.2.b. The model predictive control based in direct current control the equation for the possible predicted source current can be expressed as follows:

$i_{s, p r e}(k+1)=\frac{T_{s}}{L_{e q}}\left(V_{i}(k)-V_{s}(k)\right)+\left(1-\frac{R_{e q} T_{s}}{L_{e q}}\right) i_{s}(k)$

Finally to apply optimal switching signal to the SAPF, the all possible predicted source currents are compare with source reference current using cost function 'g'. It can as represented as

$$
g=\left(i_{s, r e f}(k+1)-i_{s, p r e}(k+1)\right)
$$

Comparing to DCC scheme for above methods the ICC scheme has the following advantages

* Using only one current sensor,

* A simple control circuit and low implementation cost.

* In a real time implementation point of view in ICC scheme used reduced number of analog signals to be read, and to avoid an additional burden of control algorithm such as subtracting filter and load current

* No delay in the compensation process

* Generation of reference current without calculating either load current or the load reactive power.

\section{Experimental results}

A single phase SAPF model is designed and analysed in MATLAB/Simulink- Simpower system toolbox. The MPC based DCC and IDCC algorithm was programmed using embedded function block and it can operated at a sampling period of $10 \mu \mathrm{s}$. The parameters used in simulation are shown in Table I, The switch on response are performed to examine performance of single phase SAPF for both MPC based DCC and IDCC scheme.

In Fig 3 to 5, illustrates the source voltage (Vs), source current (Is), load current (IL), filter current (If) and DC link capacitor voltage (Vdc) for both MPC based DCC and IDCC scheme. The simulation results shows that the switch on response and source current THD for both schemes are similar. Implementing single phase SAPF with MPC based IDCC require only one current sensor when compare to MPC based DCC scheme. 


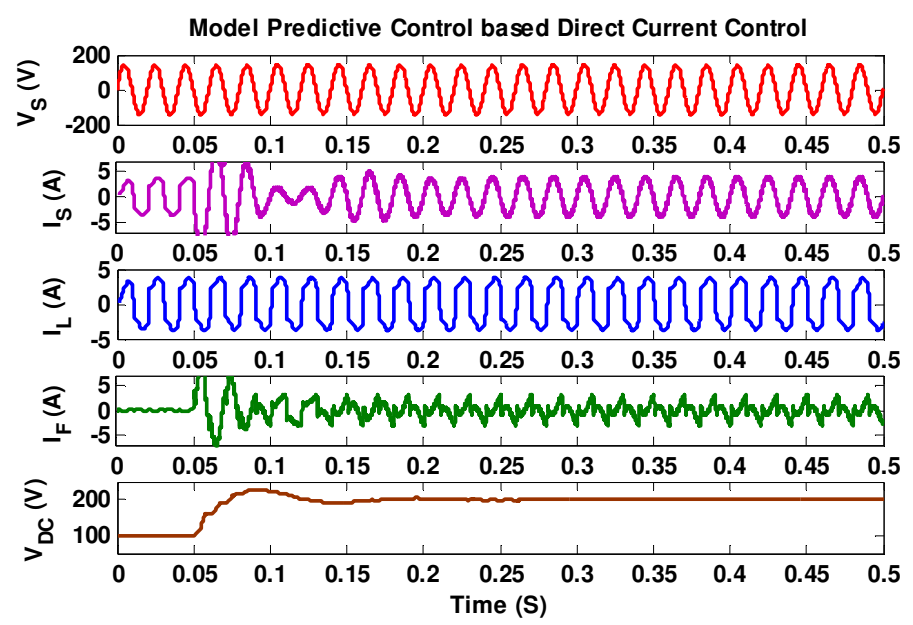

Fig. 3. MPC based DCC scheme (Switch on response)

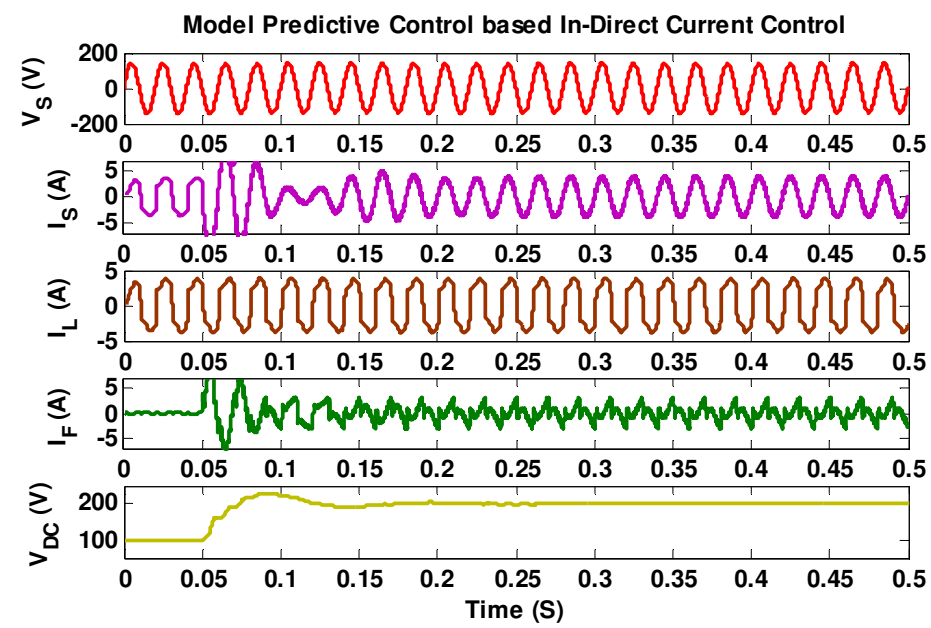

Fig. 4. MPC based IDCC scheme (Switch on response)
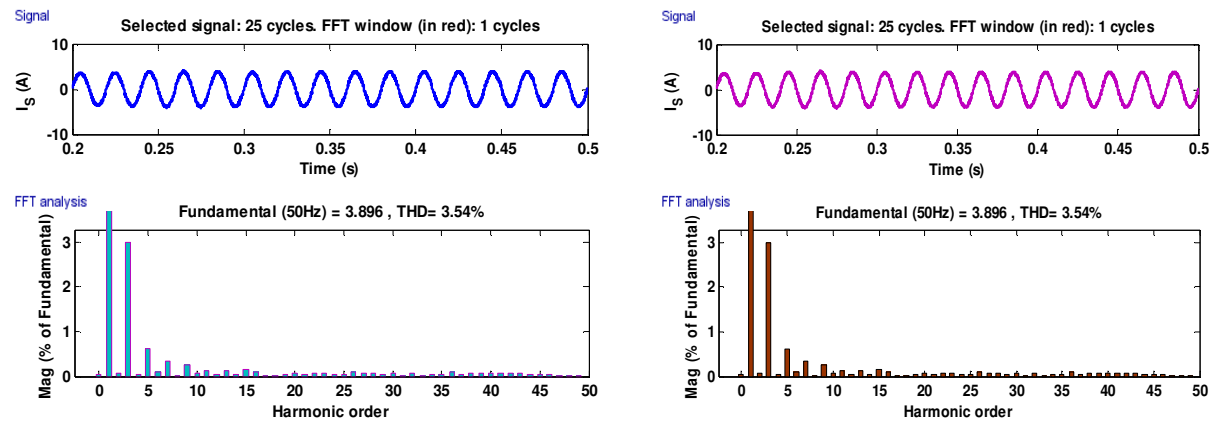

Fig 5.(a)

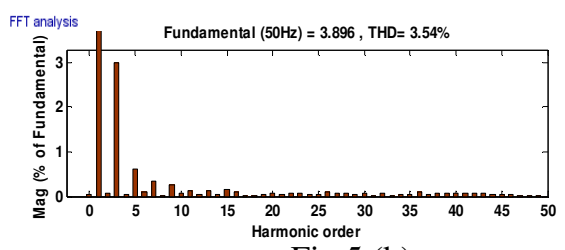

Fig.5 (b)

Fig. 5a. Harmonic spectra of simulated waveforms of single-phase SAPF with DCC scheme.

Fig. 5b. Harmonic spectra of simulated waveforms of single-phase SAPF with IDCC scheme. 
Table 1. Single phase SAPF Parameters.

\begin{tabular}{|l|c|}
\hline \multicolumn{1}{|c|}{ Parameters } & Values \\
\hline Supply voltage (Vs,rms) & $100 \mathrm{~V}$ \\
\hline DC link voltage (Vdc) & $200 \mathrm{~V}$ \\
\hline Filter resistance \& Inductance & $0.01 \Omega \& 5 \mathrm{mH}$ \\
\hline Source resistance \& Inductance & $0.1 \Omega \& 1 \mathrm{mH}$ \\
\hline MPCC control-Average Switching Frequency & $15 \mathrm{kHz}$ \\
\hline
\end{tabular}

\section{Conclusion}

The implementation of MPC based DCC and IDCC applied to single phase SAPF has been presented. A DC Link voltage control based PI control algorithm is used as harmonic extraction method to generate the reference current. When compared with MPC based DCC method, MPC based IDCC method has simple model structure, requirement of sensor is been less and easy to implement in real time.

\section{References}

1. M.V. Ataide, J.A. Pomilio, Industrial Electronics, Proceedings of the IEEE International Symposium, Vol. 2, pp.422-427, (1997).

2. S.H. Qazi, M.W. Mustafa, Renewable and Sustainable Energy Reviews, Vol. 57, pp. 420-438, (2016).

3. K. Chatterjee, B. G. Fernandes, G. K. Dubey, IEEE Transactions on Power Electronics, Vol. 2, pp.381-392, (1999).

4. F. Pottker, I. Barbi, In Power Electronics Specialists Conference, PESC'97 Record., 28th Annual IEEE, Vol. 1, pp. 412-417, (1997).

5. S. Rahmani, K. Al-Haddad, F. Fnaiech, P. Agarwal, In Industrial Electronics Society, IECON'03, The 29th Annual Conference of the IEEE, Vol.2, pp. 1044-1049, (2003).

6. R. Gupta, IEEE Transactions on Power Electronics, Vol. 5, pp. 2526-2535, (2012).

7. R. Mahanty, International Journal of Electrical Power \& Energy Systems, Vol. 62, pp. 441-449, (2014).

8. K. Rameshkumar, V. Indragandhi, K. Palanisamy, T. Arunkumari, Energy Procedia, Vol. 117, pp.658-665, (2017). 